\title{
COURRIER
}

\section{Le tabagisme, un enjeu de santé, bafoué au plus haut niveau}

Au moment où l'Europe scientifique, culturelle et politique se développe, un éditorial paru le 27 octobre 1990 dans le Lancet: "Europe for Cancer" interpelle.

Deux chiffres doivent tout d'abord être délivrés ; la Communauté Européenne consacre 5 millions de livres sterlings par an pour la campagne contre le tabagisme tandis qu'elle aide les planteurs de tabac pour 740 millions de livres sterlings annuels. Ainsi la dépense pour la prévention est-elle de 1 lorsqu'elle est de 150 pour l'extension de la culture du tabac ; n'y a-t-il pas là quelque chose de choquant lorsque l'on sait les risques du tabagisme dans trois des fléaux de l'humanité : le cancer, les maladies vasculaires, les insuffisances respiratoires.

On sait combien l'OMS a proposé l'introduction de mesures fiscales et économiques contre l'industrie du tabac permettant de réduire le tabagisme. Cette vision apparemment contraste avec celle des pays de la

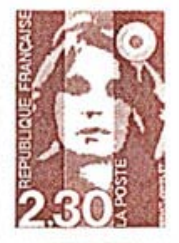

Communauté et de la Communauté elle-même, peut-être en fonction des intérêts politiques et économiques considérés à tort comme plus importants que les coûts de la santé liés au tabagisme.

Qui plus est, les Communautés Européennes aident à la culture de tabac de seconde qualité... utilisés pour intoxiquer des nations pauvres, apportant ainsi une perte de la santé pour les pays les plus défavorisés. Il faut aussi reconnaître que le tabagisme féminin croît et le risque vasculaire des enfants nés de mère tabagique est très important.

On peut considérer que l'Europe devra réduire le cancer [1] et les maladies vasculaires [2] de $15 \%$ d'ici le début du troisième millénaire. Encore faudrait-il que la Commission adopte des mesures qui puissent réduire le tabagisme et protéger la santé.

L'éditorial du Lancet finit ainsi : "Financial support for the growth of tabacco in the European region must be abolished ", je m'associe totalement à cette conclusion.

J. Caen Directeur de l'Institut des Vaisseaux et du Sang. Président d'Eurovaisseau

1. WHO EURO. Targets for health for all in the European region. Copenhagen: World health organisation, 1985.

2. Colloque Eurovaisseau, Institut de France 7-8/11/90.

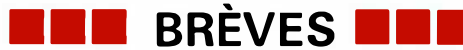

Clonage du gène et de l'ADNc de groupe A du xeroderma pigmentosum. Nous avons très récemment rapportć le clonage du gène correspondant au groupe de complémentation B du xeroderma pigmentosum, une réparatose comportant une importante hypersensibilité à la lumière solaire. Ce gène, probablement impliqué aussi dans le syndrome de Cockayne (une autre réparatose), pourrait coder pour une présomptive hélicase $\left(m / s n^{\circ} 9\right.$, vol. 6 , p. 924). C'est maintenant l'ADNc et le gène impliqués dans une forme beaucoup plus fréquente de la maladie, le groupe de complémentation A, qui vient d'être isolé par une équipe japonaise d'Osaka. La stratégie utilisée, déjà présentée dans notre $\mathrm{m} / \mathrm{s} n^{\circ} 10$, vol. 6, décembre 90 précédente nouvelle de $\mathrm{m} / \mathrm{s}$, a comporté la correction de l'hypersensibilité aux ultraviolets de cellules en culture provenant de malades atteints de xeroderma pigmentosum, groupe A, grâce au transfert d'ADN de souris. Le gène responsable de la correction a ensuite permis d'isoler les ADNc correspondants. Ceux-ci peuvent coder pour une protéine de 31000 daltons, possédant un "doigt à zinc " potentiel, et pouvant donc, présomptivement, se fixer à l'ADN. Cette protéine pourrait participer à l'une des étapes quelconques de la réparation de l'ADN endommagé. Des mutations faux-sens ou d'épissage de ce gène ont été détectées chez des malades, confirmant ainsi sa responsabilité dans cette forme de l'affection.
De plus, des anticorps développés contre la protéine recombinante et reconnaissant des protéines de 40 à $42 \mathrm{kDa}$ de poids moléculaire apparent dans les cellules normales ne détectent aucune protéine dans des cellules de malade. La différence entre le poids moléculaire déduit de la séquence de l'ADNc et celui apprécié par migration de la protéine dénaturée en gel de polyacrylamide pourrait être due à la richesse de cette protéine en prolines et à l'existence d'un domaine acide interne. Le gène, dénommé $X P A C$ (xeroderma pigmentosum group $A$ complementing), est localisé en 9q34.1.

[1. Tanaka K, et al. Nature 1990 $348: 73-6$.

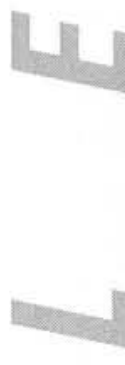

